\title{
Personalised nutrition and health
}

\author{
Jose Ordovas and colleagues consider that nutrition interventions tailored to individual \\ characteristics and behaviours have promise but more work is needed before they can deliver
}

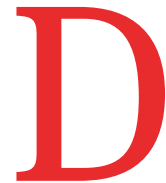

ietary factors are well recognised contributors to common diseases, including heart disease, stroke, type 2 diabetes and cancer. ${ }^{1-3}$ Despite the known link between dietary patterns and disease, interventions to alter dietary habits and to improve public health and wellbeing have had limited impact. Personalisation of interventions may be more effective in changing behaviour ${ }^{4}$ that will affect health outcomes. ${ }^{5}$ In this article we consider the evidence for personalised nutrition.

\section{What is personalised nutrition and what is it used for?}

There is no agreed definition of personalised nutrition. For the purposes of this review, we define it as an approach that uses information on individual characteristics to develop targeted nutritional advice, products, or services. Gibney et $\mathrm{al}^{6}$ describe it as an approach that "assists individuals in achieving a lasting dietary behaviour change that is beneficial for health." Personalised nutrition partially overlaps with

\section{KEY MESSAGES}

- Personalised nutrition uses informa tion on individual characteristics to develop targeted nutritional advice, products, or services to assist people to achieve a lasting dietary change in behaviour that is beneficial for health

- Personalised nutrition is based on the concept that individualised nutritional advice, products, or services will be more effective than more traditional generic approaches

- This personalisation may be based on biological evidence of differential responses to foods/nutrients dependent on genotypic or phenotypic characteristics, and/or based on current behaviour, preferences, barriers and objectives

- Most of the available evidence in support of personalised nutrition has come from observational studies with risk factors as outcomes, rather than from randomised controlled trials using clinical end points

- The overall consensus is that much research and regulation is required before personalised nutrition can deliver the expected benefits related terms such as precision nutrition, nutrigenomics, nutrigenetics, nutritional genomics, etc (box 1).

The overall goal of personalised nutrition is to preserve or increase health using genetic, phenotypic, medical, nutritional, and other relevant information about individuals to deliver more specific healthy eating guidance and other nutritional products and services (table 1). Personalised nutrition is equally applicable to patients and to healthy people who may or may not have enhanced genetic susceptibilities to specific diseases.

Personalised nutrition can be applied in two broad areas: firstly, for the dietary management of people with specific diseases or who need special nutritional support-for example, in pregnancy or old age, and, secondly, for the development of more effective interventions for improving public health. It has traditionally focused on maximising the benefits and reducing the adverse effects of dietary changes for the individual. However, this focus on the individual may have limited impact on populations. To have a wider impact, it must be deployed at a scale and in a

\section{Box 1: Descriptors and definitions}

In common with other scientific fields in their early development, multiple concepts and descriptors are used in personalised nutrition, sometimes without rigorous definition. In addition to the term personalised nutrition, many other terms are used-for example, precision nutrition, stratified nutrition, tailored nutrition, and individually tailored nutrition. We have attempted to group the descriptors as follows:

- Stratified and tailored nutrition are similar (if not synonymous). These approaches attempt to group individuals with shared characteristics and to deliver nutritional intervention/advice that is suited to each group

- Personalised nutrition and individually tailored nutrition mean similar things and go a step further by attempting to deliver nutritional intervention/advice suited to each individual

- Precision nutrition is the most ambitious of the descriptors. It suggests that it is possible to have sufficient quantitative understanding about the complex relationships between an individual, his/her food consumption, and his/her phenotype (including health) to offer nutritional intervention/advice, which is known to be individually beneficial. The degree of scientific certainty required for precision nutrition is much greater than that required for the other approaches

- Nutrigenetics is an aspect of personalised nutrition that studies the different phenotypic responses (ie, weight, blood pressure, plasma cholesterol, or glucose levels) to a specific diet (ie, low fat or Mediterranean diets), depending on the genotype of the individual

- Nutrigenomics involves the characterisation of all gene products affected by nutrients and their metabolic consequences

- Exposome is the collection of environmental factors, such as stress, physical activity and diet, to which an individual is exposed and which may affect health

As one moves from stratified to personalised to precision nutrition, it becomes necessary to apply more and more dimensions or characteristics to achieve the desired goal. For example, stratification could be undertaken using one, or a few, dimensions such as age, gender, or health status. In contrast, given the complexity of relationships between individual diet and phenotype, deployment of a wide range of dimensions/characteristics, perhaps including "big data" approaches, would be necessary to achieve the goal of precision nutrition. An exception to this broad generalisation is the management of inborn errors of metabolism such as phenylketonuria, where "precision nutrition" can be achieved using information on a single characteristic-that is, genotype.

- Epigenomics is a branch of genomics concerned with the epigenetic changes (methylation, histone modification, microRNAs) that modify the expression and function of the genetic material of an organism

- Metabolomics is the scientific study and analysis of the metabolites (usually restricted to small molecules, ie, $<900$ daltons) produced by a cell, tissue, or organism

- Microbiomics is the study of the microbiome, the totality of microbes in specific environments (ie, the human gut) 
way that reduces (rather than increases) health disparities. Individuals may also wish to use personalised nutrition to achieve personal goals/ambitions that are less directly related to health-for example, to deal with preferences for, and dislikes of, specific foods, to attempt to achieve a desired body size or shape, or for competitive sports. ${ }^{7}$

\section{What are the conceptual bases for personalised nutrition?}

Personalised nutrition is based on the idea that individualising nutritional advice, products, or services will be more effective than more generic approaches.

Personalisation can be based on:

- Biological evidence of differential responses to foods/nutrients dependent on genotypic or phenotypic characteristics

- Analysis of current behaviour, preferences, barriers, and objectives and subsequent delivery of interventions, which motivate and enable each person to make appropriate changes to his or her eating pattern.

\section{Personalisation based on biological characteristics of the individual}

Differences in the response of people to dietary components have been well documented for almost a century. ${ }^{8-10}$ This provides the basis, and motivation, for developing personalised nutrition strategies. The trend towards personalisation is the result of: firstly, nutrition research that provides a better understanding of how diet affects health; secondly, new technology that enables better and continuous measurements of markers of individual health and fitness; and thirdly, new analytical tools that interpret this flow of data and transform it into user friendly practical information. Moreover, personal nutrition integrates with the change in bioscience and public health programmes towards preventing rather than mitigating existing disease. Response to food is variable and has multiple forms. These include differential responses in plasma cholesterol concentration to dietary saturated fat intake, food allergies or intolerances (eg, lactose intolerance or gluten sensitivity), or more severe forms such as phenylketonuria and other inborn errors of metabolism. Moreover, personalised nutritional advice may be appropriate for some key factors, such as age (teenager, elderly, child, adult), stage of life (pregnant, lactating, etc), sex, BMI, disease or health status, ethnicity, and cultural or religious backgrounds that dictate particular diets

Nutrigenetics has been defined as "the discipline that studies the different phenotypic response to diet depending on the genotype of each individual." 11
It is a classic example of an attempt to characterise the response of an individual to a dietary intervention based on genetic factors. To a large extent, this is based on accumulating evidence of the phenotypic consequences of interactions between interindividual differences in genetic make up and nutrition. ${ }^{12}$ Nutrigenetics has evolved from using a unique single nucleotide polymorphism at a candidate gene locus to examine interaction with a specific nutrient (eg, saturated fat) to a more comprehensive whole genome approach analysing interactions with dietary patterns. ${ }^{13}$

More recently, new technology has enabled multiple endogenous and exogenous factors to be studied at the same time and used to predict the response to intervention. These include epigenomics, metabolomics, microbiomics (box 1), and the individual's environment, ${ }^{14}$ also known as the exposome. ${ }^{15}$ The ability to measure "everything that matters" is becoming a reality with the increasing availability of fitness trackers, mobile apps, and other devices. These enable individuals to monitor continuously multiple health related factors, such as physical activity, sleep, and vital signs-for example, blood pressure, heart rate, and stress levels. The usefulness of these devices remains controversial. ${ }^{1617}$ However, in theory, such information could be used to develop algorithms that, in combination with genetic and other biological information, may provide a sound basis for personalised recommendations.

Potentially just as important is the belief that easy access to indices of health provided frequently, and in real time, will be a driver for beneficial, and sustained behaviour change. Thus, an individual will acquire data on his/her genotype and multiple phenotypic characteristics on which the personalised nutrition is based. Periodic physiological and biochemical analyses and microbiome tests will enable tracking of their health metrics in response to dietary, and other personalised, behavioural changes in real time. Relatively little has been published on the development and validation of the algorithms for personalised nutrition. The Food4Me Study published algorithms to integrate information based on current diet, phenotypic characteristics, and genotypic characteristics. ${ }^{18}$ However, other approaches-for example, using machine-learning ${ }^{19}$ or artificial intelligence, ${ }^{20}$ might offer additional advantages.

For example, Zeevi et $\mathrm{al}^{21}$ used the connection between a raised concentration of postprandial blood glucose and the risk of type 2 diabetes risk. They monitored glucose concentrations in 800 people continuously for 1 week. They then used the variability in glycaemic response to identical test meals to devise a machinelearning algorithm that integrated blood parameters, dietary habits, anthropometrics, physical activity, and gut microbiota to predict an individual's postprandial glycaemic response to real meals. The predictive algorithm was validated in an independent cohort $(n=100)$. These investigators conducted a small randomised controlled dietary intervention study that suggested that personalised diets may successfully modify raised postprandial blood glucose.

The potential role of microbiome based information in developing personalised nutrition has been emphasised in more recent work from the same group. They used a small intervention study to show that an individual's glycaemic response to a test meal can be predicted from microbiome data before the intervention. ${ }^{22}$ These results highlight the importance of information about individual people in understanding the effects of dietary factors on metabolism and health. The results suggest that interindividual differences in responses to dietary challenges may be particularly informative, but we need evidence from larger scale studies to know whether such personalised interventions based on a "challenge test" offer significant advantages.

This approach was illustrated more recently by Price et al. ${ }^{23}$ They collected personal data, including whole genome sequences, clinical tests, blood metabolome and proteome, physical activity, and fecal microbiome, on three occasions over 9 months from 108 people. They used these data to generate correlation networks that disclosed communities of related analytes associated with physiology and disease. They also used some of the personal data (genotype and clinical markers) to implement behavioural coaching to help participants to improve biomarkers of health. This study showed, firstly, that some highly motivated people are willing to collect personal data over extended periods; secondly, that more information can help to confirm existing knowledge about the connectedness of human physiology and to expose new connections; and, thirdly, with intense measurement in highly motivated people, "personalised coaching" may help to change behaviour. However, it is not clear how much of the detailed measurement undertaken in the study was essential in developing the "personalised coaching." As the participants were self-selected, it is unclear whether this approach would be acceptable to larger populations. 


\section{Personalisation based on analysis of current behaviour, preferences, barriers, and objectives}

Most researchers, and other stakeholders in personalised nutrition, have focused on the capture of genotypic or phenotypic characteristics. The implicit assumption is that, the more we can measure, the more effective will be the outcomes of personalisation. ${ }^{24}$ There is increasing realisation that, unlike with medication, dietary changes require individuals to make daily, sometimes hourly, choices. The adoption of these lifestyle changes (including but not limited to changes in dietary patterns) is highly dependent on effective collaboration with participants who are being helped to take responsibility for their behaviour, and, ultimately, health. Increasing technology is available that can motivate healthy eating. However, such applications usually adopt a "one-size-fits-all" approach that is biased towards specific cultures or population subgroups. Evidence suggests that it is possible to facilitate a change in behaviour using genetic testing or personalised advice as the catalysts. ${ }^{25} 26$ More emphasis is needed to develop behavioural approaches that will best motivate particular individual and cultural groups.

There may be benefits in moving from a decision framework based on health professionals' perspectives of effectiveness to one of shared decision making. An intervention based on shared decision making between the provider and the recipient becomes personalised and may increase acceptance and adherence. In this regard, the Food4Me Study stands out. It was a randomised controlled trial (RCT) involving $>1600$ participants from seven European countries, which showed that personalised nutrition was more effective than a conventional one-size-fits-all approach as control (box 2). ${ }^{27} \mathrm{~A}$ limitation of the study is that no information is available on outcomes beyond 6 months. However, findings from an earlier systematic review and meta-analysis suggest that, if changes are apparent at 6 months, they are likely to be sustained for at least a year. ${ }^{28}$

None the less, many questions remain, and the conceptual framework underpinning this type of personalisation is poorly defined.

\section{Implementation challenges}

Personalised nutrition has raised expectations similar to the excitement that has surrounded other scientific developments in their early stages. Scientists working in this area have expressed concerns about overpromising, ${ }^{2930}$ individually ${ }^{3132}$ as well as through institutional guidelines and statements. ${ }^{33-38}$ Highest expectations arise from the suggestion that genetic information might be used to define personalised dietary recommendations. For example, the Academy of Nutrition and Dietetics states that "nutritional genomics provides insight into how diet and genotype interactions affect phenotype. The practical application of nutritional genomics for complex chronic disease is an emerging science and the use of nutrigenetic testing to provide dietary advice is not ready for routine dietetics practice.” The consensus is that much research

\section{Box 2: Food4Me Study}

The Food4Me Study ${ }^{27}$ is the largest randomised controlled trial to have investigated the efficacy of personalised nutrition.

The study asked two key questions:

- Is personalised nutrition more effective in changing diet than a conventional onesize-fits-all approach?

- Does the basis used for personalisation matter? (With particular interest in the benefit of personalisation based on phenotypic and genotypic characteristics)

After 6 months, the answer was clear. Personalisation of dietary advice assisted and/ or motivated consumers to eat a healthier diet and follow a healthier lifestyle (in comparison with "impersonal" (conventional) dietary advice). The Healthy Eating Index was used as the global measure of "healthfulness" of eating patterns and change was measured after 3 and 6 months.

Personalisation based on analysis of current diet was more effective in assisting and/or motivating study participants to make, and to sustain, appropriate healthy changes to their usual (habitual) diet and lifestyle. However, there was no evidence of any additional benefit from using more sophisticated, and expensive, bases for personalisation, such as phenotypic and genotypic information.

The Food4Me Study was implemented as an internet based intervention to emulate commercial personalised nutrition aids. The intervention was delivered to $>1600$ adults in seven European countries and used several new approaches to collection and validation of data and biological samples. ${ }^{47-58}$ This study provides a model for the use of the internet in delivering personalised interventions. It demonstrates the opportunities to scale up and to make potentially significant cost effective improvements in public health. is needed before personalised nutrition can deliver the expected benefits. ${ }^{36}$

Gaps in the evidence base-Firstly, most studies, many of which are nutrigenetic, have used retrospective or observational approaches. Those studies that have used interventions are small and have focused on intermediate biomarkers. Only a few reports have studied gene-diet interactions in large, randomised, long term dietary intervention studies with clinical events as endpoints. ${ }^{39} 40$ Stronger evidence for causality may come from well designed dietary RCTs that use prospective genotyping when randomising participants to treatments, as in the FINGEN Study ${ }^{41}$ (box 3). The latter study investigated the effects of supplementation with fish oil on cardiovascular risk markers. For the design and implementation of an RCT, such an approach is much less complex than trials involving whole foods or which attempt to change eating patterns. Randomised controlled trials are essential to providing proof of concept and to giving scientific credibility to the concept of personalised nutrition. We envisage that ethical providers will build delivery systems in which elements of the system are evidence based but for which it would be difficult or impossible to test the whole system with an RCT.

Applying evidence for populations to individuals-Most of our evidence in populations is probabilistic. The personalised nutrition approach wants to use this evidence for individuals. To take a simple example, there is evidence that an interaction between a variant in $A P O A 2$ and intake of saturated fatty acids has an effect on obesity and, by extrapolation, on the risk of cardiovascular disease. ${ }^{42} 43$ Lowering saturated fatty acid intake in those carrying this variant would be expected to lower obesity and thus the risk of cardiovascular disease in populations. However, for individuals, there is no guarantee of any benefit. This is because, in common with most health outcomes, the risk of cardiovascular disease is multifactorial and includes the effects of stochastic factors. Available evidence allows us to predict mean outcomes from a given intervention and genotype, but it is impossible to predict health outcomes for individuals. Thus, the current interest is in studies that measure multiple parameters at the same time. Alternatively, others have advocated single subject studies in personalised nutrition. ${ }^{44}$ Single subject, or n-of- 1 , trials can potentially assess the usefulness of personalised interventions by integrating emerging technology and biomarkers. ${ }^{45}$ Analytical approaches to $n$-of- 1 studies are being developed in related fields-for example, health 
Box 3: Personalised nutrition

\section{Strengths}

- Interindividual variability in response to dietary factors is a real phenomenon

- Some studies have shown that personalisation results in greater improvements in diet than universal approaches

- Personalisation may foster sustained change in behaviour

- The personalised nutrition approach mirrors the rise in personalised, or precision, medicine, which is likely to drive scientific developments beneficial for personalised nutrition, and, therefore, public health

Weaknesses

- Scientific evidence for personalised nutrition is mostly based on observational studies with a low level of reproducibility

- The theoretical basis for personalised nutrition is underdeveloped

- The factors responsible for interindividual differences in response to dietary factors, their persistence over time within the same individual, and their heritability are mostly unknown ${ }^{59}$

- There are few well-designed randomised controlled trial that demonstrate the efficacy and safety of personalised nutrition

- Most commercial offerings in the personalised nutrition area are based on direct to consumer tests that are unregulated and have limited published evidence of benefit

psychology, and may be suitable for use in personalised nutrition. ${ }^{46}$

Effect on health disparities-The use of most new technology (such as n-of-1 trials) for predicting and measuring the response to specific dietary changes may be prohibitively expensive if deployed at scale. ${ }^{5}$ This may increase health disparities. The challenge for research will be to define the minimum set of measurements/ biomarkers that predicts individual response to personalised nutrition.

Encouraging shared decision makingFace-to-face consultations with a health professional or lifestyle coach might enable shared decision making, but is relatively expensive. In the Food $4 \mathrm{Me}$ Study, personalisation was implemented by nutrition researchers ${ }^{52747}$ using decision trees. This guided the personalised advice and ensured that it was standardised across study sites. This process could be used to build algorithms that "tailor" the advice/ support offered to an individual, based on preferences, barriers, ambitions, etc. Such algorithms can also incorporate techniques for behaviour change to help maximise the (health) benefit. ${ }^{48}$ These algorithms could be automated and could operate in "real time" using the internet. They provide an opportunity for large scale, cost effective shared decision making that may minimise possible increases in health disparities.

\section{Is personalised nutrition more effective than alternative approaches?}

Despite studies supporting personalised nutrition, most evidence has come from observational studies with risk factors as outcomes, rather than from RCTs using clinical end points.

There are two key related questions. Firstly, can personalised nutrition produce greater, more appropriate and sustained changes in behaviour than conventional approaches? Secondly, do these changes result in better health and wellbeing?

We have limited information that the answer to the first of these questions is yes. ${ }^{2749}$ However, evidence for the usefulness of communicating genetic risks of a disease itself on risk-reducing health behaviour is weak. ${ }^{50} \mathrm{~A}$ recent systematic review studied genetic testing and lifestyle behaviour change. It concluded that behaviour change can be facilitated using genetic testing as the catalyst. The authors argued that to promote such change the theory of planned behaviour should be deployed when communicating the results of genetic testing. ${ }^{26}$

The second question remains unanswered. No personalised nutrition study has been carried out at a large scale, in an appropriate population group and over a sufficiently long time. For this reason, and because of the importance of lifestyle change for large sections of the population, other investigators advocate a universal, rather than targeted, approach to lifestyle intervention for disease prevention and treatment. ${ }^{51}$ The logistical complexity, practical challenges, and financial costs of nutrition intervention studies with disease risk as outcomes are large and likely to be increased in personalised nutrition interventions. Thus further testing will probably use outcomes such as changes in diet, adiposity, or established biomarkers of disease such as blood pressure, $\mathrm{HbA}_{1 \mathrm{c}}$, or cognitive function. In addition, there are major opportunities to test the usefulness of personalised nutrition in the response to disease management and treatment. This would be cost effective and logistically feasible.

\section{Personalised nutrition in the marketplace}

The potential market for personalised nutrition is huge. Firstly: as indicated above, it applies to both diseased and healthy people; secondly, eating is a daily activity, and thus opportunities for personalisation are continuous; thirdly, through personalisation a person may feel able to enhance or maintain health. Most commercial personalised nutrition interventions are provided directly to the consumer through the internet. The reliability of the evidence used by such companies is uncertain. ${ }^{5253}$ The business has developed without regulatory oversight, defined standards, and consumer protection. ${ }^{54}$ Moreover, there are no educational resources or guidelines for how the outcomes of research into personalised nutrition should be implemented. To protect the public, advice should be based on robust scientific evidence. A framework for testing evidence for the scientific validity of nutrigenetic knowledge has been published. ${ }^{38}$ It is intended to be used for developing transparent and scientifically sound advice to the public founded on nutrigenetic tests. This is based on the assumption that scientifically valid, properly regulated information delivered through the internet will be less expensive and more pervasive and may help to reduce health inequalities.

\section{Suggestions for the future}

Advancement of personalised nutrition will be facilitated by a number of factors. Firstly, the development of a strong theoretical basis, including identification of the most important individual characteristics on which to base personalisation. Secondly, the evidence for efficacy and cost effectiveness from well designed intervention studies. Thirdly, the introduction of a regulatory framework designed to protect the public and to give confidence to health professionals and policy makers. This will require a substantial increase in the scientific evidence. This implies:

- More robust study designs ranging from RCTs enrolling participants based on preselected genotypes, to $\mathrm{n}$-of- 1 trials and aggregated n-of-1 trials. Such research will benefit from multidisciplinary research teams, comprising, for example, behavioural psychologists, computer scientists, biomedical scientists, and nutritionists.

- Integration of other "omics" to provide greater mechanistic interpretation of the evidence. This is likely to include emphasis on epigenomics, metabolomics, and microbiomics. In this respect, proof of principle of the role of the microbiome in shaping interindividual variability in response to diet has been established.

A first step in developing guidelines for using genotype based advice in personalised nutrition has been proposed by the 
Table 1 | Different levels of recommendation for women (not pregnant or lactating)

\section{Global}

recommendation

$\mathrm{Zn}$ (8mg/day):

Consume a wide variety

of foods containing zinc

Red meat and poultry

provide the majority of

zinc in the American

diet. Other good food

sources include beans,

nuts, certain types of

seafood, whole grains,

fortified breakfast

cereals, and dairy

products
Personalised dietitian recommendation based on an individual's history

and preferences

Recommendations vary according to age, sex, pregnancy and lactation (2-13

$\mathrm{mg}$ ). Personalisation will account for these individual characteristics. In addition,

consideration should be given to:

- People who have had gastrointestinal surgery, such as weight loss surgery, or who have digestive disorders, such as ulcerative colitis or Crohn's disease. Both these conditions can decrease the amount of zinc that the body absorbs and increase the amount lost in the urine

- Vegetarians, because they do not eat meat, which is a good source of zinc Also, the beans and grains they typically eat contain compounds that prevent complete absorption of zinc by the body. For this reason, vegetarians might need to eat as much as 50\% more zinc than the recommended amounts

- Older infants who are breastfed because breast milk contains insufficient zinc for infants aged $>6$ months. Infants taking formula receive sufficient zinc. Older infants who do not take formula should be given foods that contain zinc, such as pureed meats

- Alcoholics, because alcoholic beverages decrease the amount of zinc absorbed by the body and increase the amount lost in the urine. Also, many alcoholics eat a limited amount and variety of food, so they may not get enough zinc

- People with sickle cell disease, because they might need more zinc

\section{Dietary fat and}

Use fats and oils sparingly.

cholesterol: Choose a

diet low in fat, saturated

fat, and cholesterol
Use the nutrition facts label to help you choose foods lower in fat, saturated fat, and cholestero

Eat plenty of grain products, vegetables, and fruits

Choose low fat milk products, lean meats, fish, poultry, beans, and peas to get essential nutrients without substantially increasing calories and intake of saturated fat
Personalised recommendation based on individual history, preferences, and genetic information

SLC30A8: Carriers of the A allele at the rs11558471 SLC30A8 (zinc transporter) variant need supplements containing zinc in addition to a healthy diet to maintain proper glucose homoeostasis. ${ }^{55}$ Knowledge of this genetic information will trigger a recommendation for $\mathrm{Zn}$ supplementation
Food4Me consortium. ${ }^{38}$ It will be important for research and regulatory communities to evaluate the proposed guidelines. This may lead to the development of more generic guidelines that could be valuable for national (and international) regulators. However, given the diversity of approaches to personalised nutrition, it is likely to be difficult to agree on the principles for such generic guidelines. Experience shows that commercial providers are keen to proceed before the scientific evidence is established. This would be unimportant if the commercial offerings were harmless. However, inappropriate dietary change may harm the consumer's health and finances. It will be important to find ways of curbing the more extravagant claims, which are likely to tarnish the emerging science of personalised nutrition.

Competing interests: We have read and understood BMJ policy on declaration of interests and declare the following interests: JMO is part of Habit advisory board.

Provenance and peer review: Commissioned; externally peer reviewed.

This article is one of a series commissioned by The $B M J$. Open access fees for the series were funded by Swiss Re, which had no input into the commissioning or peer review of the articles.
Jose M Ordovas, professor ${ }^{123}$

Lynnette R Ferguson, professor ${ }^{4}$

E Shyong Tai, professor ${ }^{5}$

John C Mathers, professor ${ }^{6}$

JM-USDA-HNRCA at Tufts University, Boston, MA, USA

${ }^{2}$ Centro Nacional Investigaciones Cardiovasculares,

Madrid, Spain

${ }^{3}$ IMDEA Food Institute, CEI UAM + CSIC, Madrid, Spain

${ }^{4}$ Faculty of Medical and Health Sciences, The University of Auckland, Auckland, New Zealand

${ }^{5}$ National University of Singapore, Singapore

${ }^{6}$ Human Nutrition Research Centre, Institute of Cellular Medicine, Newcastle University, Newcastle Upon Tyne, United Kingdom

Correspondence to: J M Ordovas

jose.ordovas@tufts.edu

Micha R, Peñalvo JL, Cudhea F, Imamura F, Rehm CD, Mozaffarian D. Association between dietary factors and mortality from heart disease, stroke and type 2 diabetes in the United States. JAMA 2017:317:912-24. doi:10.1001/jama.2017.0947

2 Ferlay J, Soerjomataram I, Dikshit R, et al. Cancer incidence and mortality worldwide: sources, methods and major patterns in GLOBOCAN 2012. Int J Cancer 2015:136:E359-86 doi:10.1002/ijc 29210 3 GBD 2015 Risk Factors Collaborators. Global, regional, and national comparative risk assessment of 79 behavioural, environmental and occupational, and metabolic risks or clusters of risks, 1990-2015: a systematic analysis for the Global Burden of Disease Study 2015. Lancet 2016;388:1659-724. doi:10.1016/S0140-6736(16)31679-8

4 Woolf SH, Purnell JQ. The good life: working together to promote opportunity and improve population health and well-being JAMA 2016:315:1706-8. doi:10.1001/jama.2016.4263
5 Celis-Morales C, Lara J, Mathers JC. Personalising nutritional guidance for more effective behaviour change. Proc Nutr Soc 2015;74:130-8. doi:10.1017/S0029665114001633

6 Gibney M, Walsh M, Goosens J. Personalized nutrition: paving the way to better population health. In: Eggersdorfer M, Kraemer M, Vordaro JB, et al, eds. Good nutrition: perspectives for the 21st century. Karger Publishers, 2016: 235-48.

7 Pickering C, Kiely J. Are the current guidelines on caffeine use in sport optimal for everyone? Interindividual variation in caffeine ergogenicity, and a move towards personalised sports nutrition. Sports Med 2018;48:7-16. doi:10.1007/s40279-017-0776-1

8 Miller JZ, Weinberger MH, Daugherty SA, Fineberg NS, Christian JC, Grim CE. Blood pressure response to dietary sodium restriction in healthy normotensive children. Am J Clin Nutr 1988;47:113-9. doi:10.1093/ ajcn/47.1.113

9 Morris C, O'Grada C, Ryan M, et al. Identification of differential responses to an oral glucose tolerance test in healthy adults. PLoS One 2013;8:e72890. doi:10.1371/journal.pone.0072890

10 Sweeney JS. Dietary factors that influence the dextrose tolerance test: a preliminary study. Arch Intern Med 1927;40:818-30. doi:10.1001/ archinte.1927.00130120077005

11 Corella D, Ordovas JM. Nutrigenomics in cardiovascular medicine. Circ Cardiovasc Genet 2009;2:637-51. doi:10.1161/CIRCGENETICS.109.891366

12 Corella D, Coltell O, Mattingley G, Sorlí JV, Ordovas JM. Utilizing nutritional genomics to tailor diets for the prevention of cardiovascular disease: a guide for upcoming studies and implementations. Expert Rev Mol Diagn 2017:17:495-513 doi:10.1080/14737 159.2017.1311208

13 Frazier-Wood AC. Dietary patterns, genes, and health: challenges and obstacles to be overcome. Curr Nut Rep 2015;4:82-7. doi:10.1007/s13668-014-0110-6 
14 Carlsten C, Brauer M, Brinkman F, et al. Genes, the environment and personalized medicine: we need to harness both environmental and genetic data to maximize personal and population health. EMBO Rep 2014;15:736-9. doi:10.15252/ embr.201438480

15 Siroux V, Agier L, Slama R. The exposome concept: a challenge and a potential driver for environmental health research. Eur Respir Rev 2016;25:124-9. doi:10.1183/16000617.0034-2016

16 Chiauzzi E, Rodarte C, DasMahapatra P. Patientcentered activity monitoring in the self-management of chronic health conditions. BMC Med 2015;13:77. doi:10.1186/s12916-015-0319-2

17 Patel MS, Asch DA, Volpp KG. Wearable devices as facilitators, not drivers, of health behavio change. JAMA 2015;313:459-60. doi:10.1001/ jama.2014.14781

18 Forster H, Walsh MC, O'Donovan CB, et al. A dietary feedback system for the delivery of consistent personalized dietary advice in the webbased multicenter Food4Me Study. J Med Internet Res 2016;18:e150. doi:10.2196/jmir.5620

19 Zhou M, Fukuoka Y, Mintz Y, et al. Evaluating machine learning-based automated personalized daily step goals delivered through a mobile phone app: randomized controlled trial. JMIR Mhealth Uhealth 2018;6:e28. doi:10.2196/mhealth.9117

20 Chen CH, Karvela M, Sohbati M, Shinawatra T, Toumazou C. PERSON-personalized expert recommendation system for optimized nutrition. IEEE Trans Biomed Circuits Syst 2018;12:151-60. doi:10.1109/TBCAS.2017.2760504

21 Zeevi D, Korem T, Zmora N, et al. Personalized nutrition by prediction of glycemic responses. Cell 2015;163:1079-94. doi:10.1016/j. cell.2015.11.001

22 Korem T, Zeevi D, Zmora N, et al. Bread affects clinical parameters and induces gut microbiomeassociated personal glycemic responses. Cell Metab 2017;25:1243-1253.e5. doi:10.1016/j. cmet.2017.05.002.

23 Price ND, Magis AT, Earls JC, et al. A wellness stud of 108 individuals using personal, dense, dynamic data clouds. Nat Biotechnol 2017;35:747-56. doi:10.1038/nbt.3870

24 Riedl A, Gieger C, Hauner H, Daniel H, Linseisen J. Metabotyping and its application in targeted nutrition: an overview. Br J Nutr 2017;117:1631-44. doi:10.1017/S0007114517001611

25 Macready AL, Fallaize R, Butler LT, et al. Application of behavior change techniques in a personalized nutrition electronic health intervention study: protocol for the web-based Food4Me randomized controlled trial. JMIR Res Protoc 2018;7:e87. doi:10.2196/resprot.8703

26 Horne J, Madill J, O'Connor C, Shelley J, Gilliland J. A systematic review of genetic testing and lifestyle behaviour change: are we using highquality genetic interventions and considering behaviour change theory? Lifestyle Genom 2018. doi:10.1159/000488086.

27 Celis-Morales C, Livingstone KM, Marsaux CF, et al, Food4Me Study. Effect of personalized nutrition on health-related behaviour change: evidence from the Food4Me European randomized controlled trial. Int J Epidemiol 2017;46:578-88. doi:10.1093/ije/ dyw186.

28 Lara J, Evans EH, O'Brien N, et al. Association of behaviour change techniques with effectiveness of dietary interventions among adults of retirement age: a systematic review and meta-analysis of randomised controlled trials. BMC Med 2014;12:177 doi:10.1186/s12916-014-0177-3

29 Stenne R, Hurlimann T, Godard B. Are research papers reporting results from nutrigenetics clinical research a potential source of biohype? Account Res 2012;19:285-307. doi:10.1080/08989621.20 12.718681

30 Joost HG, Gibney MJ, Cashman KD, et al. Personalised nutrition: status and perspectives.
BrJ Nutr 2007;98:26-31. doi:10.1017/ S0007114507685195

31 Stenne R, Hurlimann T, Godard B. Benefits associated with nutrigenomics research and their reporting in the scientific literature: researchers' perspectives. Account Res 2013;20:167-83. doi:10. 1080/08989621.2013.788381

32 Hurlimann T, Menuz V, Graham J, Robitaille J, Vohl MC, Godard B. Risks of nutrigenomics and nutrigenetics? What the scientists say. Genes Nutr 2014;9:370. doi:10.1007/s12263-0130370-6

33 Ramos-Lopez O, Milagro Fl, Allayee $\mathrm{H}$, et al. Guide for current nutrigenetic, nutrigenomic, and nutriepigenetic approaches for precision nutrition involving the prevention and management of chronic diseases associated with obesity. J Nutrigenet Nutrigenomics 2017;10:43-62. doi:10.1159/000477729

34 Ferguson LR, De Caterina R, Görman U, et al. Guide and position of the International Society of Nutrigenetics/Nutrigenomics on personalised nutrition: Part 1- Fields of precision nutrition. J Nutrigenet Nutrigenomics 2016;9:12-27. doi:10.1159/000445350

35 Ferguson JF, Allayee H, Gerszten RE, et al, American Heart Association Council on Functional Genomics and Translational Biology, Council on Epidemiology and Prevention, and Stroke Council. Nutrigenomics, the microbiome, and gene-environment interactions: new directions in cardiovascular disease research, prevention, and treatment: a scientific statement from the American Heart Association. Circ Cardiovasc Genet 2016;9:291-313. doi:10.1161/ HCG.0000000000000030

36 Camp KM, Trujillo E. Position of the Academy of Nutrition and Dietetics: nutritional genomics. / Acad Nutr Diet 2014;114:299-312. doi:10.1016/j. jand.2013.12.001

37 Kohlmeier M, De Caterina R, Ferguson LR, et al. Guide and position of the International Society of Nutrigenetics/Nutrigenomics on personalized nutrition: Part 2-Ethics, challenges, and endeavors of precision nutrition. J Nutrigenet Nutrigenomics 2016;9:28-46. doi:10.1159/000446347

38 Grimaldi KA, van Ommen B, Ordovas JM, et al. Proposed guidelines to evaluate scientific validity and evidence for genotype-based dietary advice. Genes Nutr 2017;12:35. doi:10.1186/s12263-0170584-0

39 Corella D, Carrasco P, Sorlí JV, et al. Mediterranean diet reduces the adverse effect of the TCF7L2 rs7903146 polymorphism on cardiovascular risk factors and stroke incidence: a randomized controlled trial in a high-cardiovascular-risk population. Diabetes Care 2013;36:3803-11. doi:10.2337/dc13-0955

40 Corella D, Asensio EM, Coltell O, et al. CLOCK gene variation is associated with incidence of type-2 diabetes and cardiovascular diseases in type-2 diabetic subjects: dietary modulation in the PREDIMED randomized trial. Cardiovasc Diabetol 2016;15:4. doi:10.1186/s12933-0150327-8

41 Caslake MJ, Miles EA, Kofler BM, et al. Effect of sex and genotype on cardiovascular biomarker response to fish oils: the FINGEN Study. Am J Clin Nutr 2008;88:618-29. doi:10.1093/ajcn/88.3.618

42 Corella D, Peloso G, Arnett DK, et al. APOA2, dietary fat, and body mass index: replication of a gene-diet interaction in 3 independent populations. Arch Intern Med 2009;169:1897-906. doi:10.1001/ archinternmed.2009.343

43 Corella D, Tai ES, Sorlí JV, et al. Association between the APOA2 promoter polymorphism and body weight in Mediterranean and Asian populations: replication of a gene-saturated fat interaction. Int J Obes (Lond) 2011;35:666-75. doi:10.1038/ijo.2010.187

44 Schork NJ, Goetz LH. Single-subject studies in translational nutrition research. Annu Rev
Nutr 2017;37:395-422. doi:10.1146/annurevnutr-071816-064717

45 Chen R, Mias GI, Li-Pook-Than J, et al. Personal omics profiling reveals dynamic molecular and medical phenotypes. Cell 2012;148:1293-307. doi:10.1016/i.cell.2012.02.009

46 Vieira R, McDonald S, Araújo-Soares V, Sniehotta $F F$, Henderson R. Dynamic modelling of $n$-of1 data: powerful and flexible data analytics applied to individualised studies. Health Psychol Rev 2017;11:222-34. doi:10.1080/17437199.201 7.1343680

47 Celis-Morales C, Livingstone KM, Woolhead C, et al. How reliable is internet-based self-reported identity, socio-demographic, and obesity measures in European adults? Genes Nutr 2015;10:28. doi:10.1007/s12263-015-0476-0

48 O'Brien N, Heaven B, Teal G, et al. Integrating evidence from systematic reviews, qualitative research, and expert knowledge using co-design techniques to develop a web-based intervention for people in the retirement transition. / Med Internet Res 2016;18:e210. doi:10.2196/jmir.5790

49 Nielsen DE, El-Sohemy A. Disclosure of genetic information and change in dietary intake: a randomized controlled trial. PLoS One 2014;9:e112665. doi:10.1371/journal. pone. 0112665

50 Hollands GJ, French DP, Griffin SJ, et al. The impact of communicating genetic risks of disease on riskreducing health behaviour: systematic review with meta-analysis. BMJ 2016;352:i1102. doi:10.1136/ bmj.i1102

51 Langenberg C, Sharp SJ, Franks PW, et al. Gene-lifestyle interaction and type 2 diabetes: the EPIC interact case-cohort study. PLoS Med 2014;11:e1001647. doi:10.1371/journal. pmed.1001647

52 Saukko P. State of play in direct-to-consumer genetic testing for lifestyle-related diseases: market, marketing content, user experiences, and regulation. Proc Nutr Soc 2013;72:53-60. doi:10.1017/ S0029665112002960

53 Guasch-Ferré M, Dashti HS, Merino J. Nutritional genomics and direct-to-consumer genetic testing: an overview. Adv Nutr 2018;9:128-35. doi:10.1093/ advances/nmy001

54 Ahlgren J, Nordgren A, Perrudin M, et al. Consumers on the internet: ethical and legal aspects of commercialization of personalized nutrition. Genes Nutr 2013;8:349-55. doi:10.1007/s12263-0130331-0

55 Kanoni S, Nettleton JA, Hivert MF, et al, MAGIC Investigators. Total zinc intake may modify the glucose-raising effect of a zinc transporter (SLC30A8) variant: a 14-cohort meta-analysis. Diabetes 2011;60:2407-16. doi:10.2337/db110176

56 McAuley E, McNulty H, Hughes C, Strain JJ, Ward M. Riboflavin status, MTHFR genotype and blood pressure: current evidence and implications for personalised nutrition. Proc Nutr Soc 2016;75:40514. doi:10.1017/S0029665116000197

57 Jaeger B, Bosch AM. Clinical presentation and outcome of riboflavin transporter deficiency: mini review after five years of experience. J Inherit Metab Dis 2016;39:559-64. doi:10.1007/s10545-0169924-2

58 Celis-Morales C, Livingstone KM, Marsaux CF, et al. Design and baseline characteristics of the Food $4 \mathrm{Me}$ study: a web-based randomised controlled trial of personalised nutrition in seven European countries. Genes Nutr 2015;10:450. doi:10.1007/s12263014-0450-2

59 Bouchard C, Tremblay A, Després JP, et al. The response to long-term overfeeding in identical twins. N Engl J Med 1990;322:1477-82. doi:10.1056/ NEJM199005243222101

Cite this as: $B M J$ 2018;361:k2173

http://dx.doi.org/10.1136/bmj.k2173 
OPEN ACCESS

This is an Open Access article distributed in accordance with the terms of the Creative Commons Attribution (CC BY 4.0) license, which permits others to distribute, remix, adapt and build upon this work, for commercial use, provided the original work is properly cited. See: http:// creativecommons.org/licenses/by/4.0/ 